\title{
ARTHRITIS BY PARACOCCIDIOIDES BRASILIENSIS IN RHEUMATOID ARTHRITIS
} Cipriano Reis Gama ${ }^{1, \star}$, Heitor Furlan Giordano ${ }^{1}$, Victor Matheus Ostrovski Souza Santos ${ }^{1}$, Ana Cristina de Medeiros Ribeiro',
Karina Rossi Bonfiglioli ${ }^{1}$

1.Universidade de São Paulo, São Paulo (SP), Brazil.

*Corresponding author: ciprianogama@gmail.com

\section{BACKGROUND}

The use of immunobiological therapy in Rheumatology has increase remission goal achievements, but target-specific treatment can also lead to higher risks for infections. Fungal arthritis caused by Paracoccidioides brasiliensis is a rare condition. There are a few reports in the literature. Cases described in rheumatoid arthritis (RA) patients are mostly associated with immunosuppression. Signs and symptoms are often insidious and subacute. When diagnosis is delayed, the outcomes can be very unfavorable, resulting in severe joint damage and compromised quality of life.

\section{CASE REPORT}

Female patient, 69 years old, long-stand RA (36 years) positives rheumatoid factor ( $25 \mathrm{IU} / \mathrm{mL}$ ) and anti-CCP (190 IU). Presenting a difficult-to-treat disease, with multiple changes in therapy due to toxicity and/or failure: recurrent infections with methotrexate, tocilizumab and infliximab (pneumonia and sinusitis), intolerance to leflunomide (headache) and sulfasalazine (gastrointestinal side effects), maculopathy secondary to hydroxychloroquine urticarial reaction to adalimumab, failure to rituximab and abatacept. She also presented chronic lung comorbidities: asthma since childhood, nonspecific interstitial pneumonitis, bronchiectasis, recurrent pneumonia, chronic use of systemic and inhaled glucocorticoids. She was using prednisone $15 \mathrm{mg} /$ day in monotherapy. The patient presented cellulitis in her left leg and monoarthritis of the ipsilateral knee associated with massive joint effusion. Diagnostic arthrocentesis was performed, and empirical use amoxicillin and clavulanic acid was started. Cellulitis had significant improvement, but not the monoarthritis. Synovial fluid analysis showed 2360 cells, lymphocytic predominance, negative for neoplastic cells, and negative cultures for aerobic, anaerobes, and mycobacteria. Culture for fungi evidenced Paracoccidioides brasiliensis after 4 weeks. Magnetic resonance imaging (MRI) of the left knee showed moderate joint effusion, marked synovitis, elongated and permeative collection with peripheral contrast enhancement in the subcutaneous layer of the anterior knee, $58.3 \mathrm{~cm}^{3}$ volume, with no signs of osteomyelitis. Treatment for fungal arthritis was started with itraconazole. After 3 months, the patient presented marked clinical improvement. Follow-up MRI showed a 90\% reduction in the collection volume and in synovitis. Another synovial fluid analysis/ culture resulted negative for fungus. Infectiology considered safe to start tofacitinib after the end of the fungal infection treatment. In 3 months, the patient presented a significant RA improvement, with a 50\% reduction in CDAI. In addition, there were no new infections.

\section{CONCLUSION}

In suspected septic arthritis, it is essential that the rheumatologist also consider a fungal etiology, especially if the patient presents subacute and monoarticular course, under immunosuppression. Early diagnosis and treatment can improve prognosis.

\section{KEYWORDS}

Rheumatoid arthritis, Fungal arthritis, Paracoccidioides brasiliensis. 